\title{
Texture Classification Based on Lacunarity Descriptors
}

\author{
João Batista Florindo and Odemir Martinez Bruno \\ Instituto de Física de São Carlos (IFSC) \\ Universidade de São Paulo (USP) \\ Avenida do Trabalhador São-carlense, 400 \\ 13560-970 São Carlos SP Brazil \\ jbflorindo@ursa.ifsc.usp.br, bruno@ifsc.usp.br
}

\begin{abstract}
The present work presents a novel solution to provide descriptors of a texture image with application in the classification of such images. The proposed method is based on the lacunarity measure of an image. We apply a multiscale transform over the power-law relation of lacunarity and extract the descriptors from a window of the multiscale transform selected whose limits are determined empirically. We compare the classification accuracy of the proposed method with other state-ofthe-art and classical texture descriptors found in the literature. We also do a brief theoretical summary of lacunarity definition, explaining its excellent performance comprobed in the results.
\end{abstract}

Keywords: Pattern Recognition, Fractal Theory, Texture Descriptors, Lacunarity.

\section{Introduction}

Nowadays, we see a growing use of fractal theory in many applied areas, such as Biology 1215], Medicine [1613], Engineering [18, among many others. Indeed, fractal theory provides a solid and rich framework for the analysis of structures presenting some kind of self-similarity patterns. This is plentifully found in natural objects and scenarios, studied in natural and physical sciences.

An interesting aspect of fractal literature is that most applications is still based only on the fractal dimension concept. Despite the fact that this metric may model many problems with a good efficiency, it still suffers from serious drawbacks, for instance, the fact that quite distinct structures may present the same fractal dimension once it follows the same self-similarity law. Another point is the questionable efficiency of a single parameter dictating the whole model of a complex system. This situation is evident in digital image analysis, the problem focused here. In this application we usually find complex patterns, often turned still more complex due to noises and other artifacts we must deal with.

Lacunarity was defined in [8] as an alternative metric for fractal objects and posteriorly generalized to other "fractal-like" structures. Roughly speaking, while fractal dimension measures the spatial filling of a fractal, lacunarity 
measures the spatial gapping of the same object. This measure is capable of distinguishing in an elegant fashion between two objects with the same fractal dimension but with different aspect. Thus, although literature still explores maidenly lacunarity concept 6510, it is a worth complement of fractal dimension as a descriptor of structures, like those present in the images analyzed here.

For the same reason mentioned as a possible cause of failure of the fractal dimension use, the use of the simple lacunarity measure may demonstrate to be inefficient in most image analysis problems. With the goal of filling this gap, we propose the development and study of a novel approach capable of providing a set of descriptors based on the lacunarity measure. This is achieved by applying a space-scale transform to the lacunarity power-law associated to the self-similar aspect of the object. In this way, we obtain a group of measures representing the lacunarity computed over different scales, emphasizing at each scale, different patterns and sub-patterns, details and irregularities. This constitutes a rich source of information about the composition and pixel distribution inside the image. Moreover, the multiscale transform binds the mathematical fractal model to the biological visual system, once such system employs widely a multiscale paradigm to extract details which will allow the discrimination of different objects.

The proposed methodology was tested in a discrimination task over the wellknown Brodatz texture image dataset 3. As waited from the theoretical context, the novel method achieved the best results when compared with classical texture descriptors methods. These results confirmed the efficiency of the proposed model as a powerful discriminator of objects even presenting a high level of complexity and noises inherent to the image generation process. Finally, the results point to the need for a deeper study of lacunarity concept in its possible applications in fractal modelling.

\section{Lacunarity}

Lacunarity is a concept defined in [8] to characterize fractal objects which despite having the same fractal dimension, present a quite dissimilar aspect, relative to their spatial distribution.

The literature shows a lot of algorithms employed in the estimation of lacunarity from the digital image representation of a shape 1. Here we apply an adaptation of gliding-box method, originally proposed in [1] to the computation of lacunarity of texture images. The idea is initially to map the gray-level representation $I:[M, N] \rightarrow \Re$ onto a surface $S$, in the following way:

$$
S=\{i, j, f(i, j) \mid(i, j) \in[1: M] \times[1: N]\},
$$

where:

$$
f(i, j)=\left\{1,2, \ldots, I_{M}\right\} \mid f=I(i, j),
$$

where $I_{M}$ is the maximum gray-level intensity present in the image. 
In the following step, we apply a threedimensional version of gliding-box algorithm to the surface. In this method, we construct a rectangular prism $R:[1: M] \times[1: N] \times\left[1: I_{M}\right]$ supporting the surface. Thus we devide such space into cubes with sidelength $r$, varying this value of $r$. For each $r$ value, we can calculate the distribution $Q(s, r)$ corresponding to the mass probability distribution:

$$
Q(s, r)=\frac{n(s, r)}{N(r)},
$$

where $n(s, r)$ is the number of boxes, with side $r$, containing $s$ points of the surface representing the object whose lacunarity we must estimate and $N(r)$ is the total number of box with side $r$. The number $s$ is also known as the mass of the box. The Figure 1 illustrates the gliding-box process.
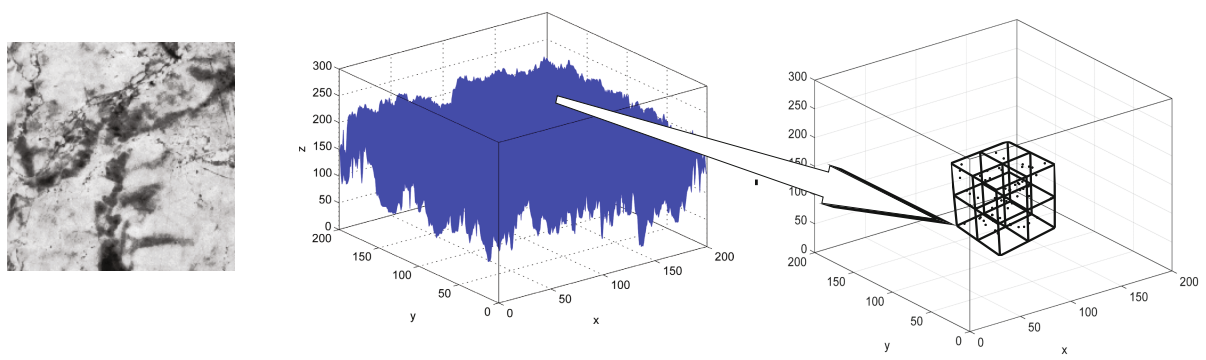

Fig. 1. Steps in the gliding-box method. From left to right, the original texture, the mapped 3D surface and the division of 3D space into cubes with sidelength $r$.

From the probability distribution, we may calculate the first and second moment $Z_{1}$ and $Z_{2}$ in a straightforward way:

$$
Z_{1}(r)=\sum_{s=1}^{s_{\max }} s Q(s, r)
$$

and

$$
Z_{2}(r)=\sum_{s=1}^{s_{\max }} s^{2} Q(s, r)
$$

From the moments we obtain the quotient $\Lambda(r)$ :

$$
\Lambda(r)=Z_{1}(r) / Z_{2}(r) .
$$

Finally, the lacunarity is calculated through the derivative:

$$
\lambda=\frac{d \log (\Lambda(r))}{d \log (r)} .
$$


In practice, the lacunarity is usually estimated by plotting the curve $\Lambda(r) \times r$ in a log-log scale and taking the slope of the straight line which may be fit to this curve.

\section{Lacunarity Texture Descriptors}

The proposed idea is based on the concept of fractal descriptors, presented in [47. Thus, the method consists in computing the lacunarity of an object, here a texture image, at different scales and taking these values to compose the object descriptors.

More formally, the values at multiple scales may be represented as a function $u(t)$ :

$$
u(t): \log (r) \rightarrow \log (\Lambda(r)),
$$

where $t$ now is the independent variable analog to $\log (r)$. In fractal descriptors approach, this function might be used diretcly or after some kind of specific post-processing depending on the particular application.

Here, in order to emphasize nuances in the function $u(t)$ which provides rich information of the texture image, we apply a multiscale transform to this function. Such kind of transform is represented by $U(b, a)$ where $b$ is related to $t$ and $a$ is the scale at which the measure is taken. In this work, we use a particular multiscale method named space-scale. This is based on the derivative of $u(t)$ followed by the convolution with a Gaussian filter aiming at attenuating possible noises emphasized by the derivative. Then, the descriptors function $\mathfrak{D}$ is provided through:

$$
\mathfrak{D}(\sigma)=\frac{d u}{d t} * g_{\sigma}(t)
$$

In this expression, $g_{\sigma}$ states for the well-known Gaussian function:

$$
g_{\sigma}(t)=\frac{1}{\sqrt{2 \pi \sigma}} \exp \left(-t^{2} / 2 \sigma\right)
$$

where $\sigma$ is the smoothing parameter.

Finally, the lacunarity descriptors are extracted from the set of values of $\mathfrak{D}$ at a specific value of $\sigma$ and thresholded at a specific point, once the last descriptors are more susceptible to noise influence. Both values of $\sigma$ and the threshold delimiter $\tau$ of the descriptors are determined empirically in each particular application. The Figure 2 shows the potential of the proposed descriptors in discriminating among texture samples from different classes.

\section{Experiments}

The experiments to verify the efficiency of the proposed technique is carried out over a classical gray-level texture dataset, namely, the Brodatz basis [3. This 

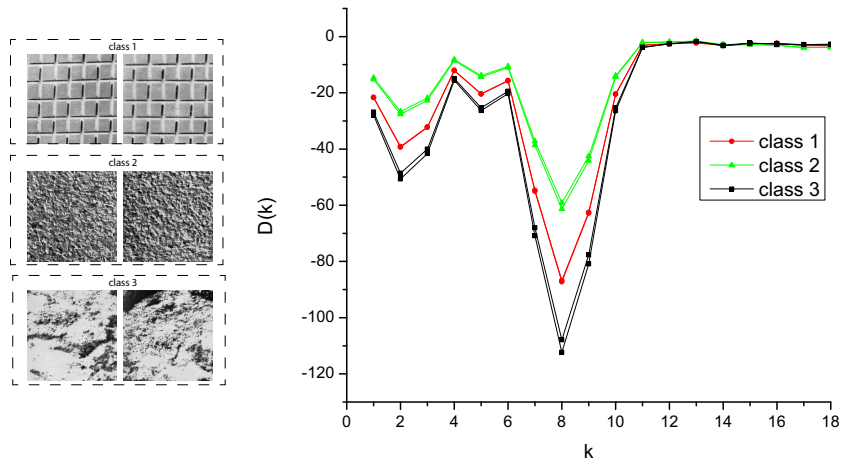

Fig. 2. Discrimination power of lacunarity descriptors. Three classes with 2 textures in each one and respective descriptors. Observe the visual distinction provided by the proposed descriptors.

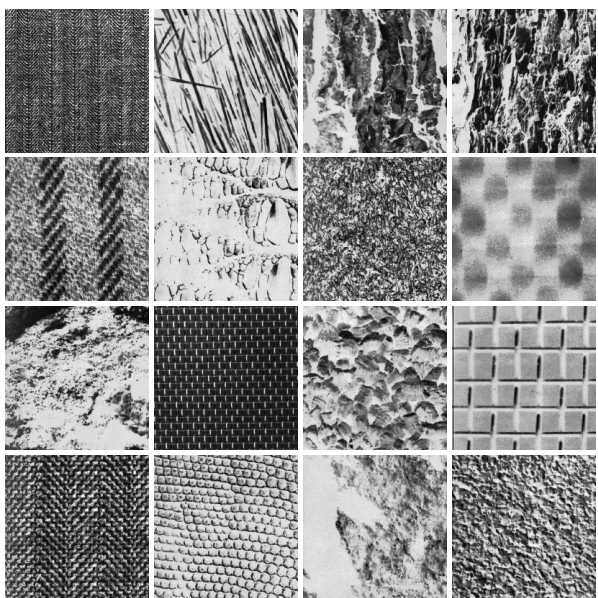

Fig. 3. Some texture samples from Brodatz dataset. Each image is from one different class.

is composed by photographs of natural scenes split into $200 \times 200$ windowed images. Each original photograph corresponds to a class and each window image to a sample. The databasis contains 111 classes with 10 samples in each one. The Figure 3 shows some image samples from the dataset.

We extract the proposed descriptors as well as other texture descriptors wellknown in the literature from each image. Beyond the lacunarity descriptors, we also applied Gabor wavelets [14], Fourier [9], Bouligand-Minkowski [2, GLDM [17] and multifractal [11 descriptors. We classify such descriptors by K-Nearest 
Neighbor classifier method, in a hold-out scheme, with $K=1$, determined empirically. Finally, we compare the classification accuracy of each tested approach.

\section{Results}

The Table 1 shows the results in terms of success rate for each compared descriptor. For the proposed method, we used $\sigma=0.1$ and a threshold $\tau=18$. We notice that lacunarity descriptors presented a significant advantage over the state-of-the-art Gabor wavelets descriptors. Another interesting point is that the proposed method uses only 18 descriptors. This reduced amount constitutes an important statistical and computational advantage once turns possible a faster computational performance and attenuates significantly any effect related to the dimensionality curse, when a large number of descriptors dissipates the discrimination ability.

Table 1. Correctness rate for Brodatz dataset

\begin{tabular}{ccc}
\hline Method & Correctness Rate (\%) & Number of descriptors \\
\hline \hline Gabor & 81.2613 & 20 \\
Fourier & 63.7838 & 74 \\
GLDM & 52.2523 & 20 \\
Multifractal & 35.1351 & 101 \\
Bouligand-Minkowski & 47.5676 & 85 \\
Proposed method & 85.5856 & 18 \\
\hline
\end{tabular}

Actually, the good performance of lacunarity descriptors was predictable, given that lacunarity is a fundamental measure associated to fractal characteristics broadly present in real-world images. As we are dealing with objects which are not real fractals strictly speaking, the lacunarity presents, in some sense, an irregular behavior along different scales. Thus, the multiscale transform highlights this aspect of imperfect power-law, providing, in this way, a valuable information of levels of lacunarity along the scales of the image. Ultimately, such lacunarity scale pattern is directly related to the psycho-visual and physical characteristics of the object represented in the texture image. So, the discrimination power is an immediate consequence of such inherent characteristics.

Finally, in the Figure 4 we show the confusion matrices for the two best methods, that is, Lacunarity and Gabor descriptors. In this figure, each point color corresponds to the number of samples pertaining to the class in vertical axis and classified as being from the class in the horizontal axis. So, the success predictions are represented in the principal diagonal while the errors are outside the diagonal. Observe that, in this case, both matrices are not so different, but the Gabor matrix shows a greater number of brighter points outside the diagonal, indicating a higher number of missclassifications, confirming the results in the Table 1 . 


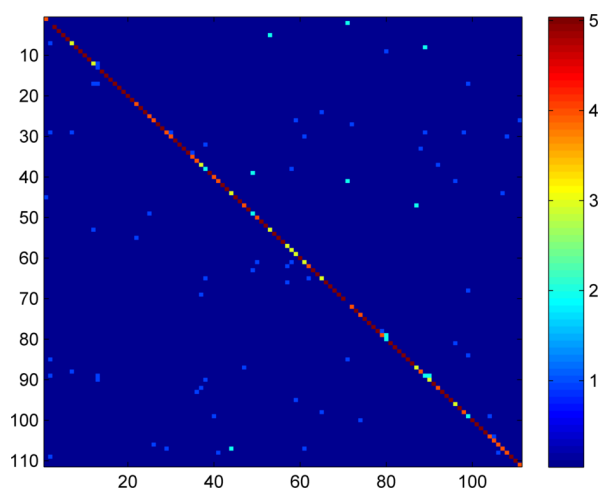

(a)

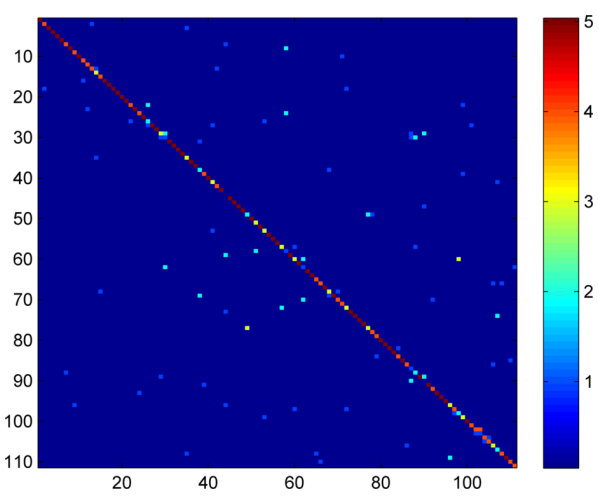

(b)

Fig. 4. Confusion matrices of the methods presenting the best performances. At left, the proposed lacunarity descriptors. At right, Gabor descriptors. Each color represent the number of samples pertaining and assigned to a class, following the colorbar notation.

\section{Conclusion}

This work proposed a novel texture descriptor technique based on the concept of lacunarity. We obtained such descriptors by applying a multiscale transform to the lacunarity computation, followed by selecting empirically a region from the multiscale response.

We compared the efficiency of the novel method with other classical and stateof-the-art texture descriptors in the classification of a benchmark dataset. The proposed descriptors presented the best performance in terms of classification accuracy. The results confirm the expectation from fractal theory. Indeed, the proposed technique demonstrates in practice that has a large potential for modelling, discriminating and describing the most complex patterns present in a real-world image.

The efficiency of the novel descriptors encourages to a deeper research for the properties of lacunarity measure in the analysis of digital images. Besides, it is comprobed that lacunarity descriptors have a large potential to be tested in applications involving image pattern recognition and computer vision in many areas of the science.

Acknowledgements. J.B.F. acknowledges support from CNPq (National Council for Scientific and Technological Development, Brazil) (Grant 140624/ 2009-0). O.M.B. acknowledges support from CNPq (Grant 308449/2010-0 and 473893/2010-0) and FAPESP (Grant 2011/01523-1). 


\section{References}

1. Allain, C., Cloitre, M.: Characterizing the lacunarity of random and deterministic fractal sets. Phys. Rev. A 44, 3552-3558 (1991)

2. Backes, A.R., Casanova, D., Bruno, O.M.: Plant leaf identification based on volumetric fractal dimension. International Journal of Pattern Recognition and Artificial Intelligence (IJPRAI) 23(6), 1145-1160 (2009)

3. Brodatz, P.: Textures: A photographic album for artists and designers. Dover Publications, New York (1966)

4. Bruno, O.M., de Oliveira Plotze, R., Falvo, M., de Castro, M.: Fractal dimension applied to plant identification. Information Sciences 178(12), 2722-2733 (2008)

5. Dong, P.: Test of a new lacunarity estimation method for image texture analysis. International Journal of Remote Sensing 21(17), 3369-3373 (2000)

6. Feagin, R.A.: Relationship of second-order lacunarity, Hurst exponent, Brownian motion, and pattern organization. Physica A: Statistical Mechanics and its Applications 328(3-4), 315-321 (2003)

7. Florindo, J.B., De Castro, M., Bruno, O.M.: Enhancing Multiscale Fractal Descriptors Using Functional Data Analysis. International Journal of Bifurcation and Chaos 20(11), 3443-3460 (2010)

8. Gefen, Y., Meir, Y., Mandelbrot, B.B., Aharony, A.: Geometric Implementation of Hypercubic Lattices with Noninteger Dimensionality by Use of Low Lacunarity Fractal Lattices. Physical Review Letters 50(3), 145+ (1983)

9. Gonzalez, R.C., Woods, R.E.: Digital Image Processing, 2nd edn. Prentice Hall, Upper Saddle River (2002)

10. Greenhill, D.R., Ripke, L.T., Hitchman, A.P., Jones, G.A., Wilkinson, G.G.: Characterization of suburban areas for land use planning using landscape ecological indicators derived from IKONOS-2 multispectral imagery. IEEE Transactions on Geoscience and Remote Sensing 41(9), 2015-2021 (2003)

11. Harte, D.: Multifractals: theory and applications. Chapman and Hall/CRC (2001)

12. Lebedev, D., Filatov, M., Kuklin, A., Islamov, A., Kentzinger, E., Pantina, R., Toperverg, B., Isaev-Ivanov, V.: Fractal nature of chromatin organization in interphase chicken erythrocyte nuclei: DNA structure exhibits biphasic fractal properties. FEBS Letters 579(6), 1465-1468 (2005)

13. Lorthois, S., Cassot, F.: Fractal analysis of vascular networks: Insights from morphogenesis. Journal of Theoretical Biology 262(4), 614-633 (2010)

14. Manjunath, B., Ma, W.: Texture features for browsing and retrieval of image data. IEEE Transactions on Pattern Analysis and Machine Intelligence 18, 837-842 (1996)

15. Quevedo, R., Jaramillo, M., Diaz, O., Pedreschi, F., Miguel Aguilera, J.: Quantification of enzymatic browning in apple slices applying the fractal texture Fourier image. Journal of Food Engineering 95(2), 285-290 (2009)

16. Tian-Gang, L., Wang, S., Zhao, N.: Fractal Research of Pathological Tissue Images. Computerized Medical Imaging and Graphics 31(8), 665-671 (2007)

17. Weszka, J., Dyer, C., Rosenfeld, A.: A comparative study of texture measures for terrain classification. SMC 6(4), 269-286 (1976)

18. Wool, R.P.: Twinkling Fractal Theory of the Glass Transition. Journal of Polymer Science Part B-Polymer Physics 46(24), 2765-2778 (2008); Annual Meeting of the American-Physical-Society, New Orleans, LA, March 10 (2008) 